\title{
PAPER
}

\section{A new theory for active suppression of reflected sound waves from the walls based on Kirchhoff-Helmholtz boundary integral equation}

\author{
Shouichi Takane* and Toshio Sone ${ }^{\dagger}$ \\ Faculty of Systems Science and Technology, Akita Prefectural University, \\ 84-4 Ebinokuchi, Tsuchiya, Honjo, 015-0055 Japan
}

(Received 12 May 2003, Accepted for publication 7 August 2003)

\begin{abstract}
In this paper, a new theory for achieving the active suppression of reflected sound waves from the walls in a room is proposed with its basis on Kirchhoff-Helmholtz boundary integral equation and inverse system theory. By actively suppressing and absorbing the unwanted reflections in the target sound field, the proposed method controls the control sources distributed outside the control region so as to equalize the region to the free field. For this purpose, Kirchhoff-Helmholtz boundary integral equation gives an effective means. Moreover, a method for suppressing the reflected sound waves from a certain part of the boundary is also mentioned. The validity of those methods is demonstrated via computer simulations.
\end{abstract}

Keywords: Active suppression, Reflected sound waves, Kirchhoff-Helmholtz boundary integral equation, Inverse system theory, Equalization, Free field

PACS number: 43.50.Gf, 43.60.Sx [DOI: 10.1250/ast.25.37]

\section{INTRODUCTION}

Active absorption of sound in a room is one of the important techniques for realizing the free field condition especially in low frequency region where the passive method is little effective. Moreover, it is expected for such a technique to be effectively applied to the absorption of a certain part of sound energy, such as the suppression of the reflected sound waves from the walls in a room.

Approaches to active suppression of reflected waves in sound field can be divided into two classes. One is for making the control sound sources which absorb the energy of the reflected sound waves. As a classical work, Olson et al. proposed the theory of "Electronic sound absorber," and its effectiveness was experimentally shown in 1953 [1]. Although the frequency range where the absorber was operated effectively was limited below $400 \mathrm{~Hz}$ because of the limitation due to the microphone and loudspeaker used at that time, this might be the first developped active absorber. In the paper by Olson et al., they mentioned, as one of the application, the absorbers located in the corner of a room for the active suppression of modes generated in a room. Studies on active sound absorber came out from 1970 's with the development of hardwares relating to it.

\footnotetext{
*e-mail: takane@akita-pu.ac.jp

$\dagger$ e-mail: tsone@akita-pu.ac.jp
}

Most of those studies are intended to actively absorb the reflected component in a duct. For example, Kido proposed the non-reflective termination of a duct by active means [2]. Ise et al. applied adaptive signal processing to the active absorption of the sound in a duct [3]. Guicking et al. directly extended the approach to one-dimensional sound field to three-dimensional one $[4,5]$. In order to apply this approach to more practical use, Nishimura et al. considered the division of the wall into small elements as compared with the wavelength of sound under control, and they installed "AAT-Cell" (Active Acoustic Treatment) to each of these elements [6]. Each AAT-Cell works independently so as to control the acoustic impedance on the cell surface to $\rho c$, where $\rho$ and $c$ are a density of air and speed of sound in air, respectively. This means that the acoustic impedance on the cell surface is turned into the acoustic impedance equal to that for plane wave.

In the works mentioned above, active control of acoustic impedance or sound intensity was attempted. Acoustic impedance is sometimes related to the sound reflection and absorption coefficient, respectively defined as $R$ and $\alpha$. If the acoustic impedance on the cell surface is expressed by $Z, R$ and $\alpha$ are respectively expressed as $R=$ $(Z-\rho c) /(Z+\rho c)$ and $\alpha=1-|R|^{2}$. If $Z$ is equal to $\rho c$, then $R=0$ and $\alpha=1$ are derived. This indicates absolute absorption with no reflection. It should be noted, however, that these relations stand only in one-dimensional sound 
field. In three-dimensional sound field, active control of the acoustic impedance of the wall into $\rho c$ does not necessarily mean the perfect suppression of reflected sound waves from it.

The other approach is based on equalizing the relevant sound field to that of the free field. Ise showed the principle of sound field control based on the Kirchhoff-Helmholtz boundary integral equation and the inverse system theory $[7,8]$. Since this approach generally requires multiple sensors for observing sound pressure and its derivatives, control sources and transfer functions from sensors to the sources, the system for implementation of this approach tends inevitably to be large. However, the crucial difference of this approach from the former one is that the free field condition can be achieved if the control sources work as expected.

This principle, however, is not valid if the primary sound source is inside the relevant sound field. Considering the active suppression of the reflected sound waves in a room with the primary sound sources at arbitrary positions, this may be the serious drawback. In this paper, a new theory for active suppression of reflected sound waves from the walls in a room including the primary sound sources inside the relevant sound field is proposed based on the Kirchhoff-Helmholtz integral equation and the theory of the inverse system. Moreover, simple computer simulations are executed in order to demonstrate the effectiveness of the proposed theory.

\section{PRINCIPLES}

\subsection{Kirchhoff-Helmholtz Boundary Integral Equation in the Free Field}

Sound pressure $P(\boldsymbol{r}, \omega)$ at an arbitrary point $\boldsymbol{r}$ in the sound field can be expressed by the sum of the incident component $P_{\mathrm{I}}(\boldsymbol{r}, \omega)$ and the reflected one $P_{\mathrm{R}}(\boldsymbol{r}, \omega)$ as:

$$
P(\boldsymbol{r}, \omega)=P_{\mathrm{I}}(\boldsymbol{r}, \omega)+P_{\mathrm{R}}(\boldsymbol{r}, \omega),
$$

where $\omega$ denotes the angular frequency of sound. As shown in Fig. 1, the sound field in the enclosed region $\Omega$ with its boundary $\Gamma$ can be represented by the KirchhoffHelmholtz boundary integral equation as follows:

$$
\begin{aligned}
P\left(\boldsymbol{r}_{P}, \omega\right)=P_{\mathrm{I}}\left(\boldsymbol{r}_{P}, \omega\right) & +\int_{\Gamma\left(\boldsymbol{r}_{q}\right)}\left\{G_{\mathrm{F}}\left(\boldsymbol{r}_{P}, \boldsymbol{r}_{q}, \omega\right) \frac{\partial P\left(\boldsymbol{r}_{q}, \omega\right)}{\partial n_{q}}\right. \\
& \left.-P\left(\boldsymbol{r}_{q}, \omega\right) \frac{\partial G_{\mathrm{F}}\left(\boldsymbol{r}_{P}, \boldsymbol{r}_{q}, \omega\right)}{\partial n_{q}}\right\} d \Gamma,
\end{aligned}
$$

where $\boldsymbol{r}_{P}$ represents the position vector of an arbitrary point in $\Omega$, and $G_{\mathrm{F}}\left(\boldsymbol{r}_{P}, \boldsymbol{r}_{q}, \omega\right)$ is the Green function of the Helmholtz equation. When $P_{\mathrm{I}}(\boldsymbol{r}, \omega)=0, i$. e. no primary source exists inside $\Omega$, only the boundary integral term is on the right hand side of Eq. (2). This integral term consists of the sound pressure, the Green function and their

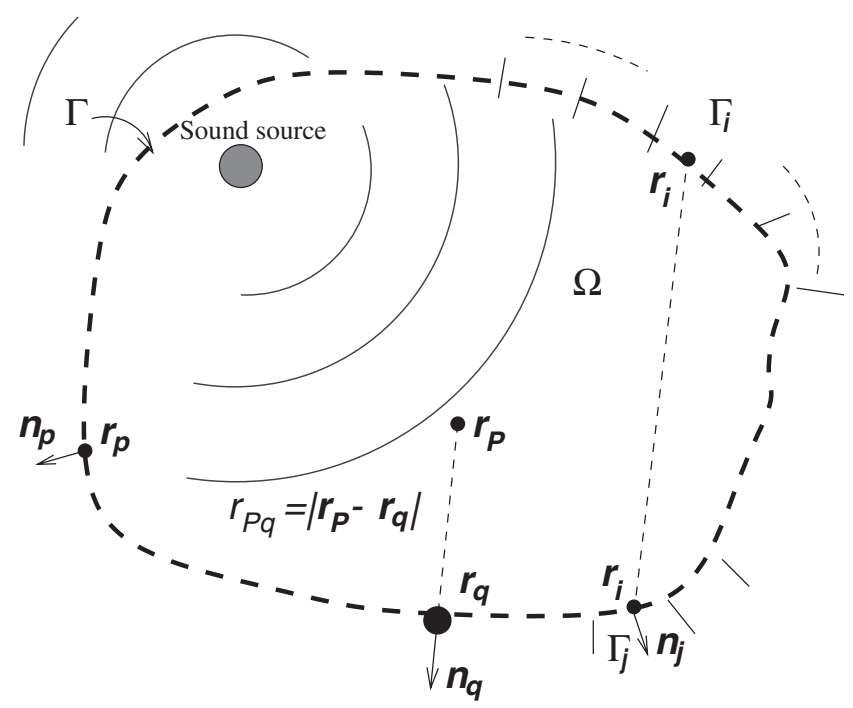

Fig. 1 Illustration of the target sound field $\Omega$ with the primary sound source in it.

derivatives on $\Gamma$. This indicates that the sound pressure at arbitrary point $\boldsymbol{r}_{P}$ is reproduced if the sound pressure and its derivative on the boundary $\Gamma$ are given. Based on this principle, Ise proposed the theory of sound field reproduction and the inverse system theory $[7,8]$. If the original sound field is the free field, the sound field inside $\Omega$ is equalized to the free field according to this principle.

However, when $P_{\mathrm{I}}(\boldsymbol{r}, \omega) \neq 0$, i.e. the primary sources exist inside $\Omega$, the direct component from these sources remains on the right hand side of Eq. (2). In this case, the free field is no longer reproduced even if the the sound pressure and its derivative on $\Gamma$ are equalized to those of the free field. Meanwhile, if the equalization of the sound field to the free field is attempted, the Kirchhoff-Helmholtz boundary integral equation has another useful property. When any acoustical obstacle does not exist inside and outside $\Omega$, i.e., the sound field is free field, the following equation is obviously valid:

$$
P\left(\boldsymbol{r}_{P}, \omega\right)=P_{\mathrm{I}}\left(\boldsymbol{r}_{P}, \omega\right) .
$$

Hence the following equation is obtained for any arbitrary point $\boldsymbol{r}_{P} \in \Omega$ :

$$
\begin{aligned}
& \int_{\Gamma\left(\boldsymbol{r}_{q}\right)}\left\{G_{\mathrm{F}}\left(\boldsymbol{r}_{P}, \boldsymbol{r}_{q}, \omega\right) \frac{\partial P\left(\boldsymbol{r}_{q}, \omega\right)}{\partial n_{q}}\right. \\
& \left.-P\left(\boldsymbol{r}_{q}, \omega\right) \frac{\partial G_{\mathrm{F}}\left(\boldsymbol{r}_{P}, \boldsymbol{r}_{q}, \omega\right)}{\partial n_{q}}\right\} d \Gamma=0 .
\end{aligned}
$$

\subsection{Another Meaning of Boundary Integral}

The condition for equalizing the sound field in $\Omega$ to free field denoted as Eq. (3) provides another meaning if the domain of integration is changed from the whole boundary $\Gamma$ to a certain part of the boundary $\Gamma_{i}$, for 
example, as shown in Fig. 1. In other words, the following boundary integral

$$
\begin{aligned}
\int_{\Gamma_{i}\left(\boldsymbol{r}_{q}\right)} & \left\{G_{\mathrm{F}}\left(\boldsymbol{r}_{P}, \boldsymbol{r}_{q}, \omega\right) \frac{\partial P\left(\boldsymbol{r}_{q}, \omega\right)}{\partial n_{q}}\right. \\
- & \left.P\left(\boldsymbol{r}_{q}, \omega\right) \frac{\partial G_{\mathrm{F}}\left(\boldsymbol{r}_{P}, \boldsymbol{r}_{q}, \omega\right)}{\partial n_{q}}\right\} d \Gamma,
\end{aligned}
$$

indicates the contribution of $\Gamma_{i}$ to the sound pressure at $\boldsymbol{r}_{P} \in \Omega$. If Eq. (4) is controlled to be zero, it means that the contribution of $\Gamma_{i}$ to the sound pressure at $\boldsymbol{r}_{P} \in \Omega$ is eliminated. This idea is useful for the suppression of reflected sound waves from a specific part of the wall.

\subsection{Proposed Control Systems}

Based on the principle mentioned in sections 2.1 and 2.2 , two types of control systems can be developed. They are respectively called "Global Suppression (GS)" and "Local Suppression (LS)."

\subsubsection{Global Suppression (GS)}

The system for the implementation of Global Suppression (GS) is illustrated in Fig. 2. In the target sound field with its boundary represented by the dashed line, Eq. (2) is valid. Since the sound field depicted in Fig. 2 is not free field, Eq. (3) is not valid. In order to produce the free field in $\Omega$, the secondary (control) sources are located outside $\Omega$, and the output from them are controlled so as to realize the condition represented by Eq. (3). When the control sources are drived, sound pressure at the arbitrary point $\boldsymbol{r}$ is expressed as follows:

$$
P(\boldsymbol{r}, \omega)=P_{\mathrm{I}}(\boldsymbol{r}, \omega)+P_{\mathrm{R}}(\boldsymbol{r}, \omega)+\sum_{j=1}^{M} H_{j}(\boldsymbol{r}, \omega) X_{j}(\omega),
$$

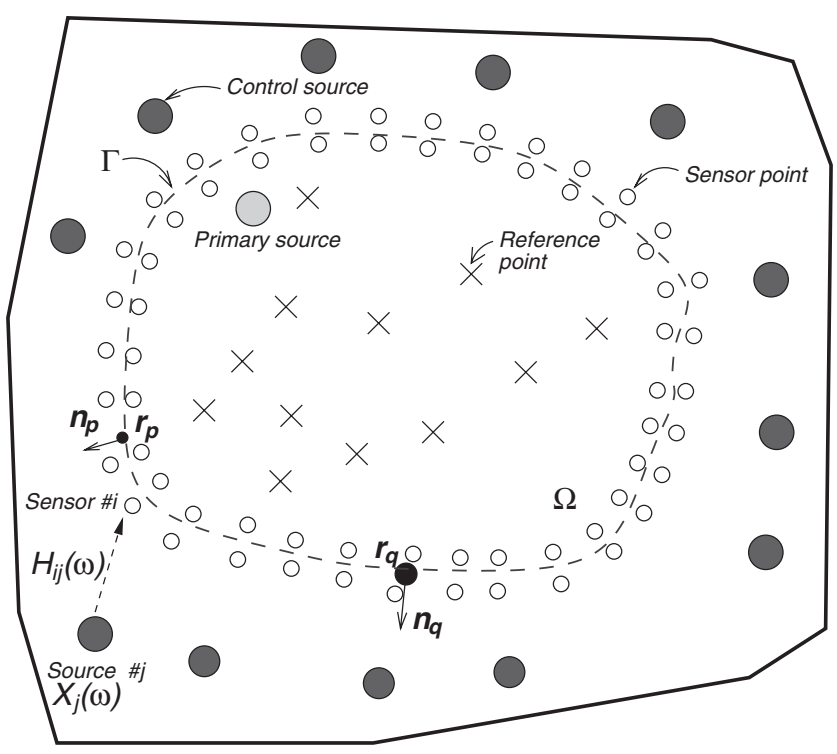

Fig. 2 The control system for the Global Suppression (GS). where $M$ indicates the number of control sources, $H_{j}(\boldsymbol{r}, \omega)$ denotes the transfer function from the $j$-th control source to the point $r$, and $X_{j}(\omega)$ is the input to the $j$-th control source. In order to calculate the left hand side of Eq. (3), the reference points (corresponding to $\boldsymbol{r}_{P}$ in Eq. (3) are chosen in $\Omega$, and Eq. (3) is computed at each reference point. $X_{j}(\omega)$ is determined by substituting Eq. (5) for Eq. (3).

When the boundary $\Gamma$ is divided into $N$ elements $\Gamma_{i}$ $(i=1, \cdots, N)$ which are assumed to be small enough comparing to the wavelength of sound under control, the sound pressure and its derivative are assumed to be constant on each element. Under this condition, Eq. (3) is approximated as follows:

$$
\sum_{i=1}^{N}\left\{A_{i}\left(\boldsymbol{r}^{(l)}, \omega\right) \frac{\partial P\left(\boldsymbol{r}_{i}, \omega\right)}{\partial n_{i}}-B_{i}\left(\boldsymbol{r}^{(l)}, \omega\right) P\left(\boldsymbol{r}_{i}, \omega\right)\right\} \approx 0,
$$

where

$$
\begin{aligned}
& A_{i}(\boldsymbol{r}, \omega)=\int_{\Gamma_{i}\left(\boldsymbol{r}_{i}\right)} G_{\mathrm{F}}\left(\boldsymbol{r}, \boldsymbol{r}_{i}, \omega\right) d \Gamma, \\
& B_{i}(\boldsymbol{r}, \omega)=\int_{\Gamma_{i}\left(\boldsymbol{r}_{i}\right)} \frac{\partial G_{\mathrm{F}}\left(\boldsymbol{r}, \boldsymbol{r}_{i}, \omega\right)}{\partial n_{i}} d \Gamma,
\end{aligned}
$$

and $\boldsymbol{r}^{(l)}(l=1, \cdots, L)$ denotes a position of $l$-th reference point in $\Omega$. Moreover, when the derivatives of the sound pressure and the Green function on the boundary $\Gamma_{i}$ are approximated by their differences between two close points $\boldsymbol{r}_{i}^{+}, \boldsymbol{r}_{i}{ }^{-}$of which interval between them is expressed by $\delta_{i}$, Eq. (6) is further modified as follows:

$$
\sum_{i=1}^{N} C_{i}\left\{\Delta P\left(\boldsymbol{r}_{i}, \omega\right) G_{i l}(\omega)-P\left(\boldsymbol{r}_{i}, \omega\right) \Delta G_{i l}(\omega)\right\} \approx 0,
$$

where

$$
\begin{aligned}
C_{i} & =\frac{\Delta S_{i}}{\delta_{i}}, \\
\Delta P\left(\boldsymbol{r}_{i}, \omega\right) & =P\left(\boldsymbol{r}_{i}^{+}, \omega\right)-P\left(\boldsymbol{r}_{i}^{-}, \omega\right), \\
G_{i l}(\omega) & =G_{\mathrm{F}}\left(\boldsymbol{r}^{(l)}, \boldsymbol{r}_{i}, \omega\right), \\
\Delta G_{i l}(\omega) & =G_{i l}^{+}(\omega)-G_{i l}^{-}(\omega), \\
G_{i l}^{+}(\omega) & =G_{\mathrm{F}}\left(\boldsymbol{r}^{(l)}, \boldsymbol{r}_{i}^{+}, \omega\right), \\
G_{i l}^{-}(\omega) & =G_{\mathrm{F}}\left(\boldsymbol{r}^{(l)}, \boldsymbol{r}_{i}^{-}, \omega\right),
\end{aligned}
$$

and $\Delta S_{i}$ indicates the area of the $i$-th element $\Gamma_{i}$. Substitution of $P\left(\boldsymbol{r}_{i}, \omega\right), P\left(\boldsymbol{r}_{i}^{+}, \omega\right)$ and $P\left(\boldsymbol{r}_{i}^{-}, \omega\right)$ for Eq. (5) gives the following simultaneous equations:

$$
D x=-f \text {. }
$$

The vector $\boldsymbol{x}$ has the strength of the control sources $X_{j}(\omega)$, and the matrix $\boldsymbol{D}$ and the vector $\boldsymbol{f}$ respectively have the components as the followings:

$D_{l j}(\omega)=\sum_{i=1}^{N} C_{i}\left\{\Delta H_{i j}(\omega) G_{i l}(\omega)-H_{i j}(\omega) \Delta G_{i l}(\omega)\right\}$ 
$F_{l}(\omega)=\sum_{i=1}^{N} C_{i}\left\{\Delta P_{0}\left(\boldsymbol{r}_{i}, \omega\right) G_{i l}(\omega)-P_{0}\left(\boldsymbol{r}_{i}, \omega\right) \Delta G_{i l}(\omega)\right\}$

where

$$
\begin{aligned}
\Delta H_{i j}(\omega) & =H_{i j}^{+}(\omega)-H_{i j}^{-}(\omega), \\
H_{i j}(\omega) & =H_{j}\left(\boldsymbol{r}_{i}, \omega\right), \\
H_{i j}^{+}(\omega) & =H_{j}\left(\boldsymbol{r}_{i}^{+}, \omega\right), \\
H_{i j}^{-}(\omega) & =H_{j}\left(\boldsymbol{r}_{i}^{-}, \omega\right), \\
P_{0}\left(\boldsymbol{r}_{i}, \omega\right) & =P_{\mathrm{I}}\left(\boldsymbol{r}_{i}, \omega\right)+P_{\mathrm{R}}\left(\boldsymbol{r}_{i}, \omega\right), \\
\Delta P_{0}(\omega) & =P_{0}\left(\boldsymbol{r}_{i}^{+} \omega\right)-P_{0}\left(\boldsymbol{r}_{i}^{-}, \omega\right),
\end{aligned}
$$

Dimensions of $\boldsymbol{D}, \boldsymbol{x}$ and $\boldsymbol{f}$ are $L \times M, M$ and $L$, respectively, and $P_{0}(\boldsymbol{r}, \omega)$ in Eq. (18) indicates the sound pressure at $r$ when the control sources are not driven. $P_{0}(\boldsymbol{r}, \omega)$ and $\Delta P_{0}(\boldsymbol{r}, \omega)$ can be observed on $\Gamma$ only when the control is off. Hence it seems to force the on-line control impossible unless the transfer functions from the primary sources to the sensor points are obtained before the control. This is a serious drawback in implementation of the active suppression of the reflected sound waves according to the proposed theory.

In order to resolve this problem, one possible way is to obtain the transfer functions from the primary sources to the sensor points. If the number of the primary source is one, $P_{0}(\boldsymbol{r}, \omega)$ and $\Delta P_{0}(\boldsymbol{r}, \omega)$ are explained by using the transfer functions as follows:

$$
\begin{gathered}
P_{0}\left(\boldsymbol{r}_{i}, \omega\right)=E_{i}(\omega) X_{0}(\omega), \\
P_{0}\left(\boldsymbol{r}_{i}^{+}, \omega\right)=E_{i}^{+}(\omega) X_{0}(\omega), \\
P_{0}\left(\boldsymbol{r}_{i}^{-}, \omega\right)=E_{i}^{-}(\omega) X_{0}(\omega),
\end{gathered}
$$

where $X_{0}(\omega)$ is the input signal to the primary source, $E_{i}(\omega), E_{i}^{+}(\omega)$ and $E_{i}^{-}(\omega)$ are the transfer functions from the primary source to the sensor point at $\boldsymbol{r}_{i}, \boldsymbol{r}_{i}^{+}$and $\boldsymbol{r}_{i}^{-}$, respectively. From Eqs. (25) (27), Eq. (16) and Eq. (18) can be modified as

$$
\boldsymbol{D} \boldsymbol{x}=-\boldsymbol{y} X_{0}(\omega),
$$

and the component of the vector $\boldsymbol{y}$ is

$$
Y_{l}(\omega)=\sum_{i=1}^{N} C_{i}\left\{\Delta E_{i}(\omega) G_{i l}(\omega)-E_{i}(\omega) \Delta G_{i l}(\omega)\right\},
$$

where

$$
\Delta E_{i}(\omega)=E_{i}^{+}(\omega)-E_{i}^{-}(\omega) .
$$

Furthermore, when the generalized inverse matrix of $\boldsymbol{D}$, denoted as $\boldsymbol{D}^{*}$, is calculated before the control works, the vector of the strengths of the control sources $\boldsymbol{x}$ may effectively be calculated as

$$
\boldsymbol{x}=-\boldsymbol{D}^{*} \boldsymbol{y} X_{0}(\omega)
$$

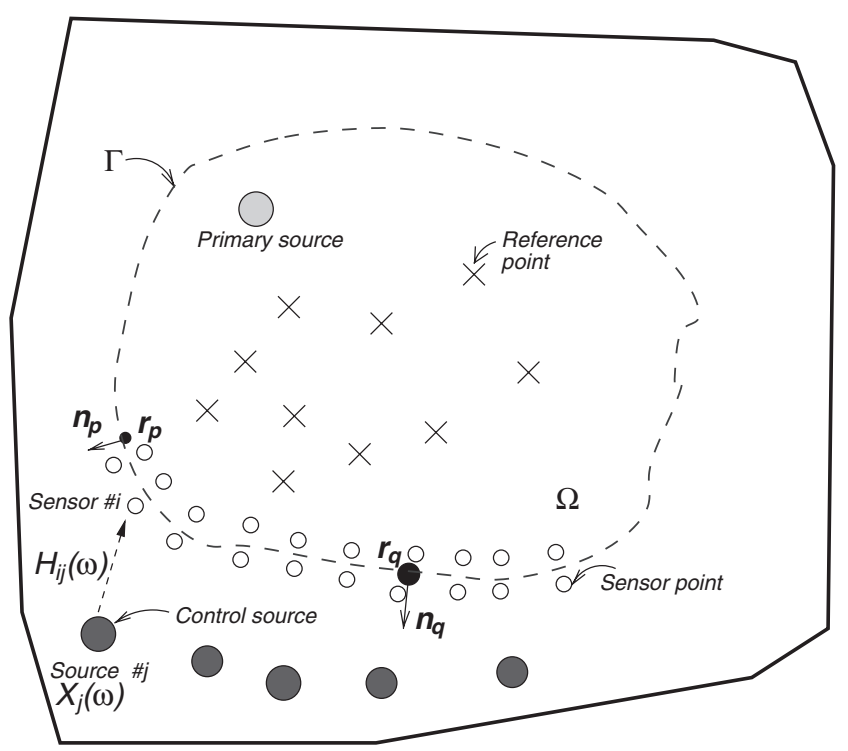

Fig. 3 The control system for the Local Suppression (LS).

Another way is to model the sound field with the Finite Element Method (FEM) and the state feedback control. Samejima et al. executed active mimimization of the sound energy in the enclosure by means of these methods [9], and Takahashi et al. applied this approach to the active suppression of the reflected sound waves [10].

\subsubsection{Local Suppression (LS)}

The system for the implementation of Local Suppression (LS) is illustrated in Fig. 3. The main differences from Fig. 2 are that the sensor points are not on the whole boundary $\Gamma$ but on a part of $\Gamma$, and that the number of the control sources drawn in Fig. 3 is reduced. Strength of each control source is determined by the same procedure as mentioned in section 2.3.1. Positions of the sensor point depend on the results of the control, $i$. $e$. from what part of the contribution to the sound pressure at the reference points is intended to be eliminated, while there exists no restriction on the position of the control sources.

\subsection{Relation of the Proposed Theory to the Preceding Study by Mangiante}

As a study relevant to this paper, Mangiante discussed active sound absorption based on Huygen's principle, and executed some experiments using his developped "ANA (Active Noise Absorber)" [11]. Since the KirchhoffHelmholtz boundary integral equation is a variational expression of the Huygen's principle [12], Mangiante's study is regarded as similar to ours with the same theoretical background. However, in his study, sound pressure and its derivative on the boundary are directly controlled by locating the monopole and dipole sources on it, while the control sources are located outside the control region in our proposed theory. The theory of inverse system plays the effective role in making the control 


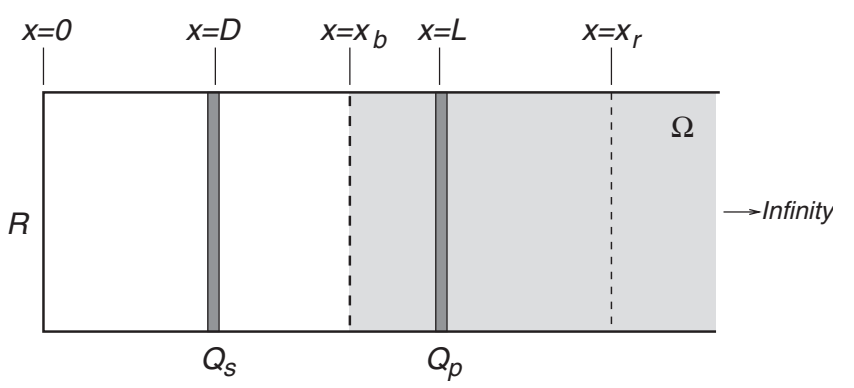

Fig. 4 Sketch of the one-dimensional sound field.

possible for such a configuration. Moreover, the ANA was proposed so that the sound propagating into the boundary of ANA is absorbed, thus the suppression of the reflected sound waves with ANA may be a matter of the configuration. Our proposal is intended to suppress the reflected sound waves, and to keep the direct component as far as possible.

\section{FUNDAMENTAL DISCUSSION IN ONE-DIMENSIONAL SOUND FIELD}

In order to show the validity of the proposed theory, the theory is applied to one-dimensional sound field as the simplest case. Sketch of one-dimnensional sound field is depicted in Fig. 4. Reflective termination is at $x=0$, having the reflection coefficient of $R(\omega)$. Primary source of strength $Q_{\mathrm{p}}$ is located at $x=L$, and the strength of the secondary (control) source $Q_{\mathrm{s}}$ is determined so as to minimize the reflection from the termination to the region $\Omega$. The control source is assumed to be located at $x=D$.

In this case, the sound field generated by the primary and the control sources are expressed respectively as the following equations for $x>L$ :

$$
\begin{aligned}
& P_{\mathrm{p}}(x, \omega)=\frac{\rho c}{2 S} Q_{\mathrm{p}}\left\{e^{-j k(L-x)}+R(\omega) e^{-j k(x+L)}\right\}, \\
& P_{\mathrm{s}}(x, \omega)=\frac{\rho c}{2 S} Q_{\mathrm{s}}\left\{e^{-j k(x-D)}+R(\omega) e^{-j k(x+D)}\right\},
\end{aligned}
$$

where $\rho, c$ are the density of air and the speed of sound in air, respectively, and $S$ is the area of the intersection. From Eqs. (32) and (33), the value of $Q_{\mathrm{s}}$ that eliminates the second term in the right hand side of Eq. (32) is derived as follows:

$$
Q_{\mathrm{s}}=\frac{-R(\omega) e^{-j k L}}{e^{j k D}+R(\omega) e^{-j k D}} Q_{\mathrm{p}}
$$

On the other hand, $Q_{\mathrm{s}}$ is calculated according to the proposed theory, defining the boundary at $x=$ $x_{\mathrm{b}}\left(D<x_{\mathrm{b}}<L\right)$. The Green function in one-dimensional sound field is expressed as

$$
G_{1}\left(x_{1}, x_{2}, \omega\right)=\frac{-j}{2 k} e^{-j k\left|x_{1}-x_{2}\right|}
$$

and $P(x, \omega)=P_{\mathrm{p}}(x, \omega)+P_{\mathrm{s}}(x, \omega)$. When Eq. (3) is calcu- lated with locating the reference point at $x=x_{\mathrm{r}}\left(x_{\mathrm{r}}>x_{\mathrm{b}}\right)$, the following equation is induced:

$G_{1}\left(x_{\mathrm{b}}, x_{\mathrm{r}}, \omega\right) \frac{\partial P\left(x_{\mathrm{b}}, \omega\right)}{\partial x_{\mathrm{b}}}-\frac{\partial G_{1}\left(x_{\mathrm{b}}, x_{\mathrm{r}}, \omega\right)}{\partial x_{\mathrm{b}}} P\left(x_{\mathrm{b}}, \omega\right)=0$.

Substitution of $G_{1}\left(x_{\mathrm{b}}, x_{\mathrm{r}}, \omega\right)$ in Eq. (36) for Eq. (35) gives

$$
R(\omega) e^{-j k L} Q_{\mathrm{p}}+\left\{e^{j k D}+R(\omega) e^{-j k D}\right\} Q_{\mathrm{s}}=0 .
$$

Obviously the same relation as Eq. (34) is derived. This indicates that the optimum strength of the control source can be derived according to the proposed principle for onedimensional sound field.

\section{COMPUTER SIMULATIONS}

As examples demonstrating the validity of the proposed principle, computer simulations were executed for two dimensional sound field.

\subsection{Computer Simulation of GS}

4.1.1. Conditions of the computer simulation

Illustration of the control of GS is shown in Fig. 5. The control region is a rectangle with its size of $2.6 \mathrm{~m} \times 1.8 \mathrm{~m}$. A rigid wall is at $y=0$, and a primary source is located at the center of the control region. The control sources are installed around the control region with approximately the same intervals. 26 reference points are located in the control region with almost the same intervals, too. The sensor point has also the same interval for each other, located on the boundary of the rectangular control region, drawn with a broken line. Frequency range of the control is $100 \sim 500 \mathrm{~Hz}$.

\subsubsection{Results and discussion}

As examples, Fig. 6(a) indicates the equal sound pressure level contour in the frequency of $200 \mathrm{~Hz}$ when the control is off, whereas Fig. 6(b) shows that when the control is on with 14 control sources. $*, \bigcirc$ and + in Fig. 6

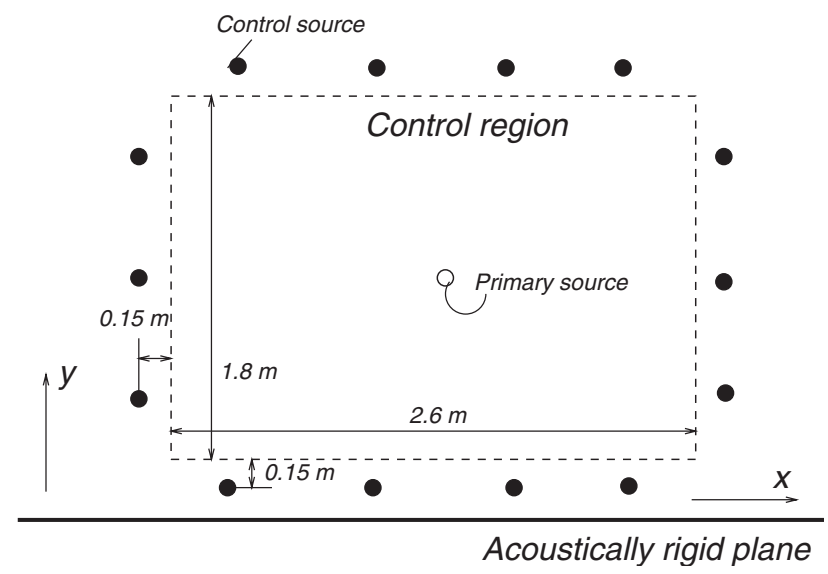

Fig. 5 Two-dimensional sound field with a single rigid wall used for computer simulation of Global Suppression. 


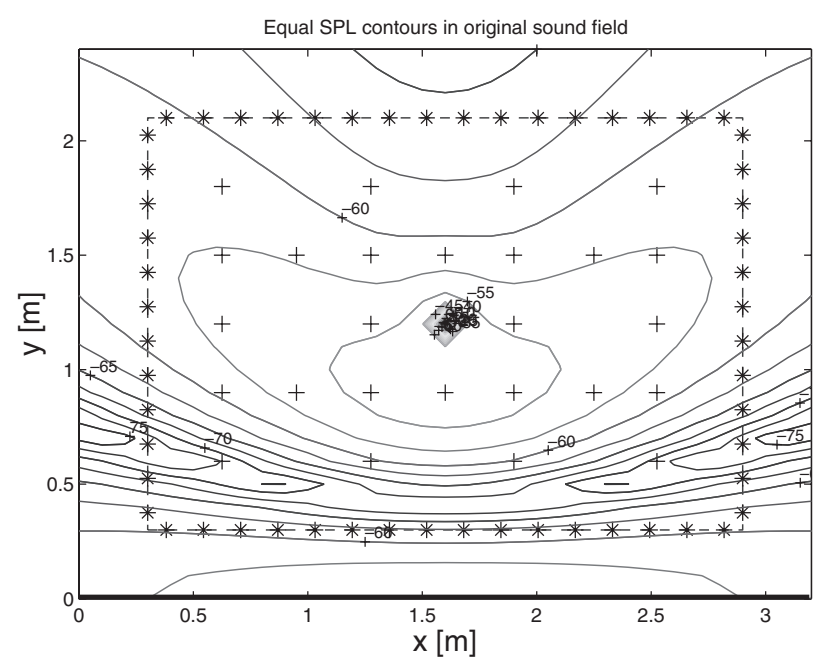

(a) Control off

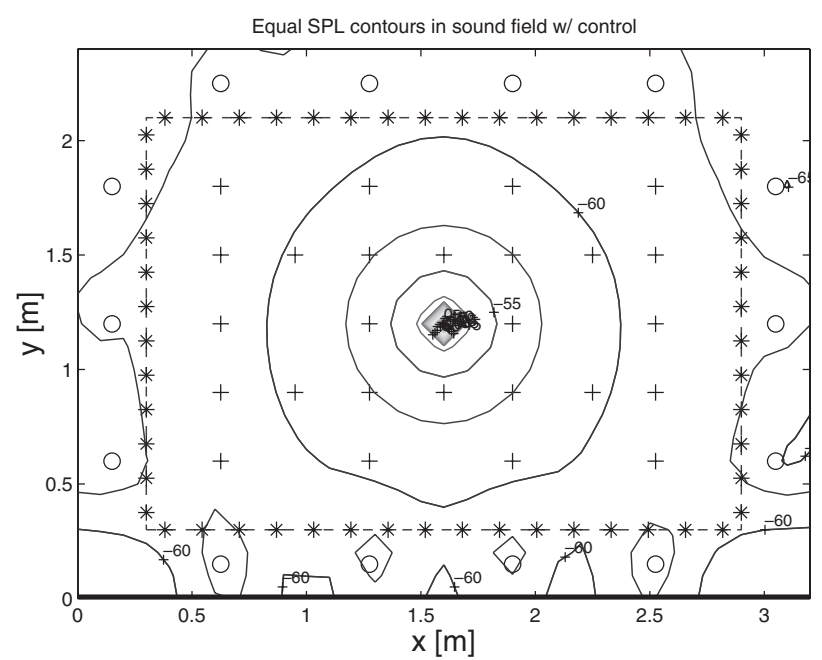

(b) Control on

Fig. 6 Equal sound pressure level contours in twodimensional sound field with a single rigid plane (Frequency: $200 \mathrm{~Hz}$, number of control sources: 14).

denote the sensor point, control source, and reference point, respectively. It is clearly seen in Fig. 6(b) that the sound pressure distribution produced by the sound wave radiated from the primary source is revived in the target sound field. Fig. 7 shows the frequency characteristics of the average error level in the control region, which is defined as follows:

$$
E_{g}=20 \log _{10} \iint_{\Omega}\left|\frac{P(\boldsymbol{r}, \omega)-P_{\mathrm{I}}(\boldsymbol{r}, \omega)}{P_{\mathrm{I}}(\boldsymbol{r}, \omega)}\right| d \Omega \quad[\mathrm{dB}]
$$

In the frequency range less than about $250 \mathrm{~Hz}$, the average error level is almost less than $-20 \mathrm{~dB}$, but when the frequency is higher, the average error level becomes larger. In the condition of the simulation used here, the intervals of sensor points on the boundary was about $0.15 \mathrm{~m}$, and the wavelength of the $400 \mathrm{~Hz}$ sound in air was taken as about $0.8 \mathrm{~m}$. Thus the interval of the sensor points is considered to be short enough, nonetheless the performance of the

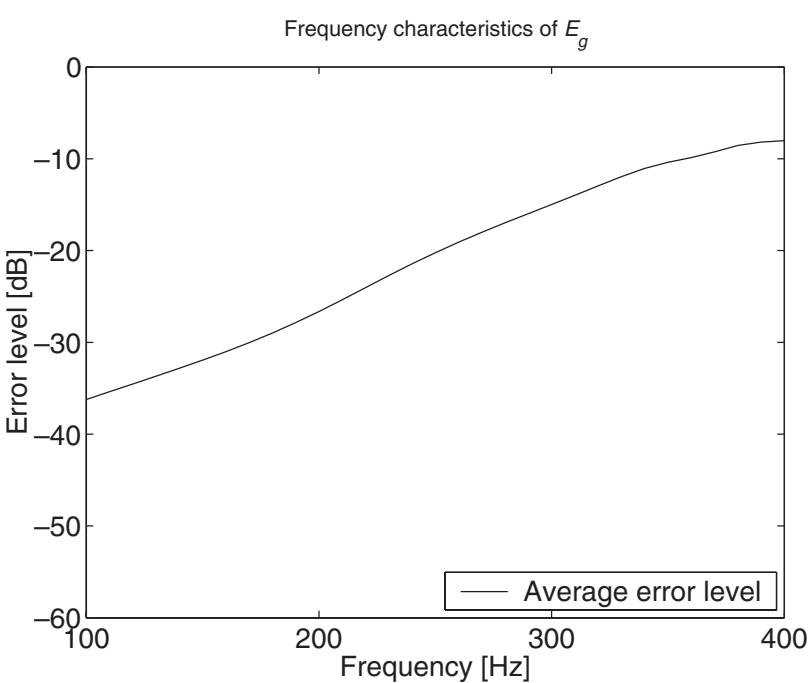

Fig. 7 Frequency characteristics of the average error level in the control region.

control with the proposed method is not good. The position of the control sources and reference points may affect the performance of the proposed method. Investigation of this will be the future works.

\subsection{Computer Simulation of LS}

\subsubsection{Conditions of the computer simulation}

Setup for the computer simulation of the LS is shown in Fig. 8. The main differences from the sound field in Fig. 5 are the positions of control sources and sensor points. They are parallel to the rigid wall at $y=0$, and symmetrically located for the point of the primary sound source. In this configuration, the reflected sound wave propagates from the vicinity of the place where the sensors and the control sources are located. If the sound propagating from this part

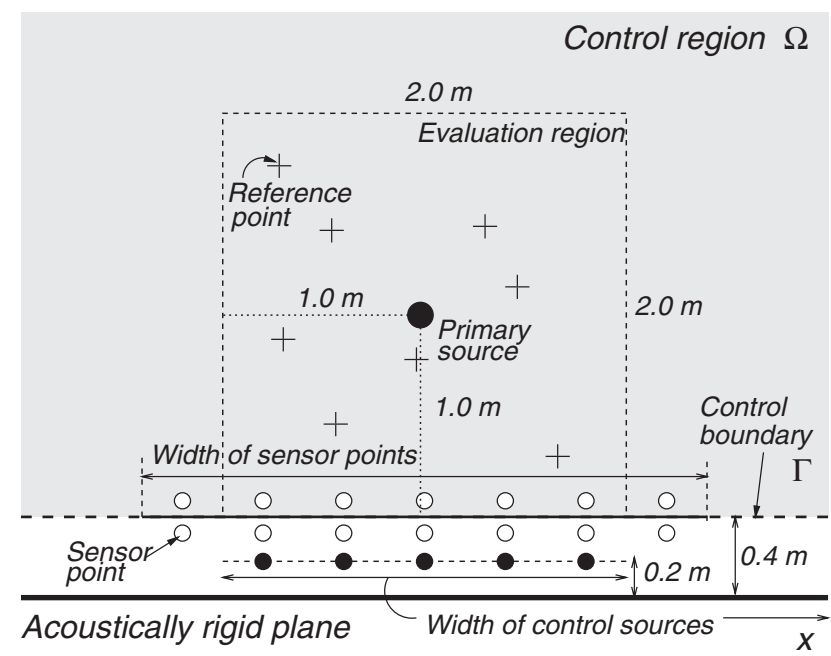

Fig. 8 Two-dimensional sound field with a single rigid wall used for computer simulation of Local Suppression. 
is suppressed, the reflected component may almost be eliminated in the region enclosed by dashed line in Fig. 8. 25 reference points are located with the same intervals inside the region.

\subsubsection{Results and discussion}

Figure 9 shows the equal sound pressure level contours when the control is on at the source frequency of $200 \mathrm{~Hz}$ with various number of control sources. Fig. 9(a) shows the result when the number of control sources is 2, and Fig. 9(b) shows that when the number is 5. All symbols in this figure are the same as those in Fig. 6. It can be seen that the sound pressure in the region is almost circular, which indicates the reflected sound from the wall is sufficiently suppressed.

In the configuration of this computer simulation, small number of the control sources is enough for the suppression of the reflection from a single rigid wall. These results are considered to be obtained for the reason that the reflection from the part of the wall, where the control sources are located around, mainly contributes to the resultant reflected sound waves. Further investigation is required on the effect of the positioning of the control sources, sensor points, and the reference points.

\section{CONCLUDING REMARKS}

In this paper, a new theory for the active suppression of reflected sound waves from the walls in a room is proposed. This theory is expressed as the free field equalization of the relevant sound field, and the condition for the control is derived from the Kirchhoff-Helmholtz boundary integral equation. It was also mentioned that the boundary integral of a certain part of the boundary is regarded as the contribution of the sound propagating from that part to the sound pressure inside the control region, and the minimization of that boundary integral corresponds to the minimization of that contribution. Based on the proposed theory, two types of control named "Global Suppression (GS)" and "Local Suppression (LS)" were explained.

In order to show the validity of the proposed theory, the elementary discussion in one-dimensional sound field was first executed. As a result, it was shown that the strength of the control source derived from the proposed theory is equal to the optimum solution. The computer simulations in two-dimensional sound field were executed. The results of the computer simulations showed that the reflected sound wave from the wall is suppressed in both GS and LS.

It is suggested from the results of the computer simulation that the future works are required for the effect of control parameters, such as position and number of the reference points, sensor points and control sources.

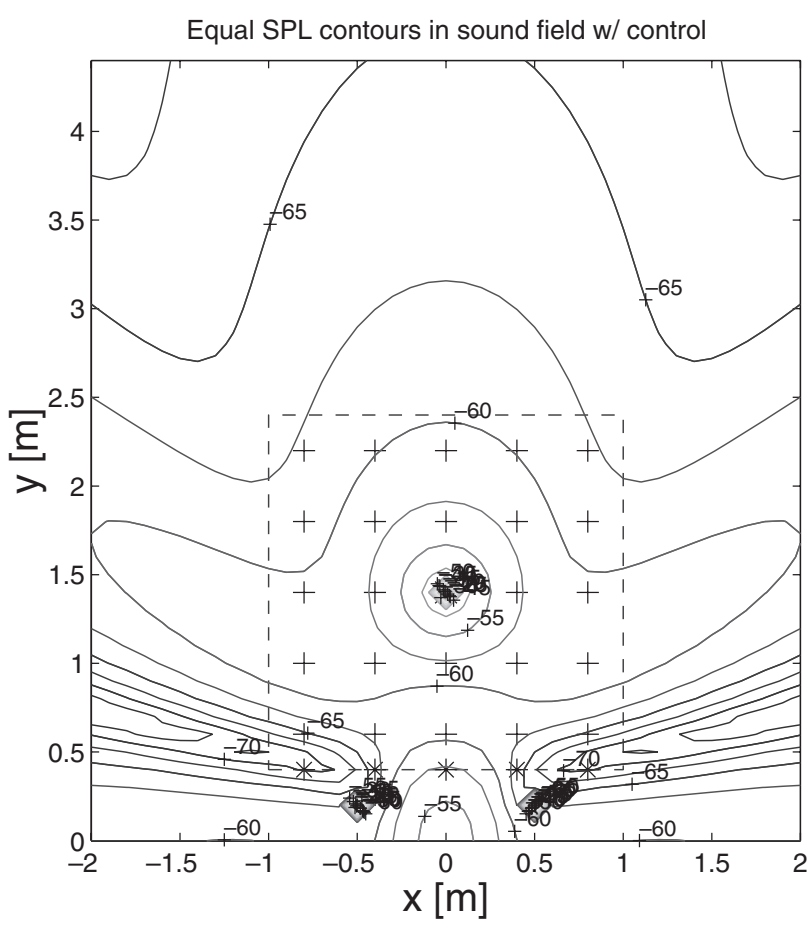

(a) No. of control sources: 2

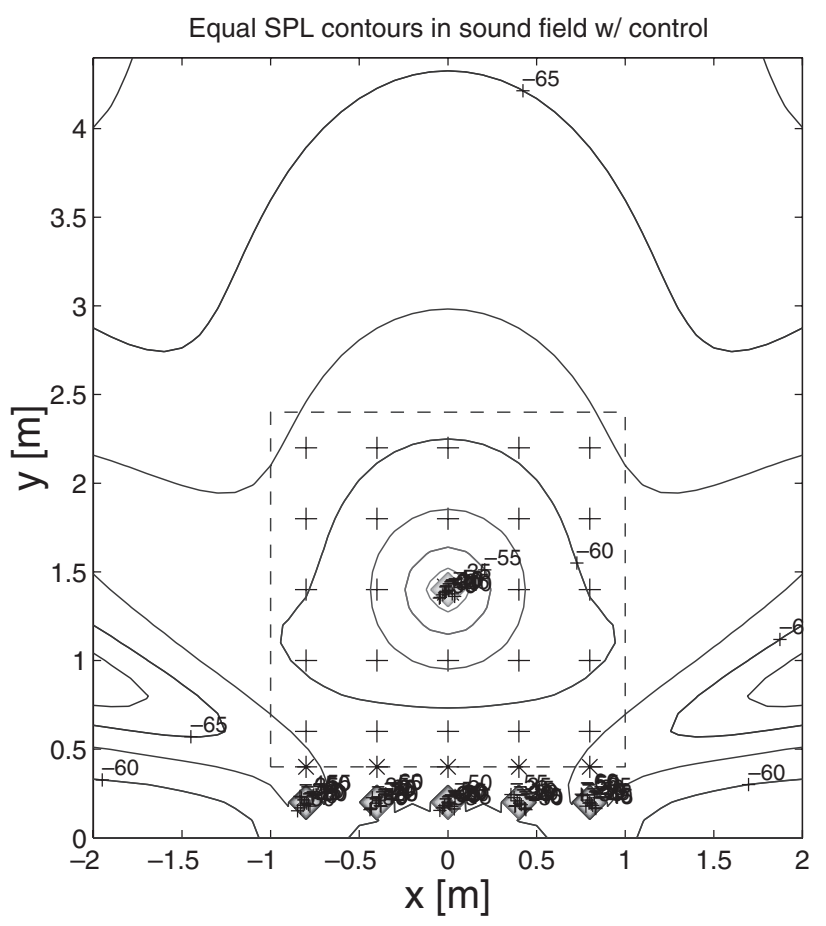

(b) No. of control sources: 5

Fig. 9 Equal sound pressure level contours in twodimensional sound field with a single rigid plane and various number of control sources (Frequency: $200 \mathrm{~Hz})$.

\section{REFERENCES}

[1] H. F. Olson and E. G. May, "Electronic sound absorber," J. Acoust. Soc. Am., 25, 1130-1136 (1953).

[2] K. Kido, "Reduction on noise by use of additional sound sources," Proc. Inter-Noise 75, pp. 64-65 (1975). 
[3] S. Ise and H. Tachibana, "Active sound absorber using adaptive signal processing," J. Acoust. Soc. Jpn. (E), 17, 305 310 (1996).

[4] D. Guicking and E. Lorenz, "Active impedance control for one-dimensional sound," ASME J. Vib. Acoust. Stress Reliab. Des., 106, 393-396 (1984).

[5] D. Guicking, K. Karcher and M. Rollwage, "Coherent active methods for applications in room acoustics," J. Acoust. Soc. Am., 78, 1426-1434 (1985).

[6] M. Nishimura, K. Ohnishi, W. P. Patrick and A. C. Zander, "Development of active acoustic treatment (phase 1)," Proc. Active 97 (1997).

[7] S. Ise, "Theory of the sound field reproduction based on the Kirchhoff-Helmholtz integral equation," Proc. 15th ICA, Vol. I, pp. 85-88 (1995).

[8] S. Ise, "A principle of sound field control based on the Kirchhoff-Helmholtz integral equation and the theory of inverse system," Acustica - Acta Acustica, 85, 78-87 (1999).

[9] T. Samejima and M. Yasuoka, "Active suppression of reverberation in a sound field through state feedback control," J. Acoust. Soc. Jpn. (J), 55, 12-22 (1999).

[10] H. Takahashi, Y. Suzuki, S. Takane and F. Asano, "Active control of sound intensity for suppression of reflected sound waves based on the state feedback control," IEICE Trans. Fundam., E84-A, 1017-1026 (2001).

[11] G. A. Mangiante, "Active sound absorption," J. Acoust. Soc. Am., 61, 1516-1523 (1977).

[12] P. A. Nelson and S. J. Elliott, Active Control of Sound (Academic Press, London, 1992), pp. 282-284.

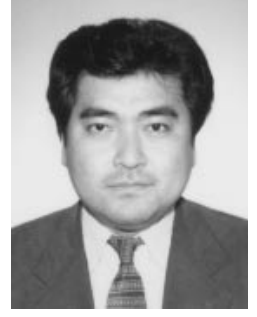

Shouichi Takane was born in Tokyo, Japan in 1967. He received B. E., M. E. , and Ph. D. degrees from Tohoku University in 1989, 1991 and 1998, respectively. He is currently an associate professor at the Faculty of Systems Science and Technology, Akita Prefectural University. His research interests include highdefinition acoustic display, sound field control, numerical analysis of sound field and acoustic signal processing. He is a member of the Acoustical Society of Japan, the Virtual Reality Society of Japan, the Institute of Electronics, Information and Communication Engineers, and other societies.

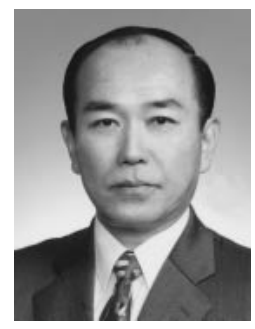

Toshio Sone graduated from Tohoku University in 1958 and received his $\mathrm{Ph}$. D. degree in electrical and communication engineering in 1963. He was a professor of Tohoku University until 1999 and is now a professor of the Faculty of Systems Science and Technology, Akita Prefectural University. He has been engaged in researches on psychological acoustics, electroacoustics, room acoustics, and noise control for more than 40 years. $\mathrm{He}$ is a former president of the Acoustical Society of Japan and a fellow of the Acoustical Society of America, a member of the Institute of Noise Control Engineering/Japan, a member of the Engineering Academy of Japan and other societies. 